\title{
GLUTATHIONE AND SELENIUM AS CHEMOPREVENTIVE AGENTS ON EXPERIMENTALLY INDUCED HAMSTER BUCCAL POUCH EPITHELIAL DYSPLASIA
}

\author{
Kamal Abd Elrahman Kamal ${ }^{*}$, Amr Saad AbdoAlwahab * and Abd Elnasser Abd El mola Esmail ${ }^{* *}$
}

\begin{abstract}
The aim of the present study was directed to investigate the effect of Glutathione (GSH) and Selenium (Se) as chemopreventive agents in experimentally induced hamster buccal pouch (HBP) epithelial dysplasia.

Material and methods: Twenty five golden Syrian male hamsters were used as experimental animals and divided into five groups (G1, G2, G3, G4 and G5): G1: 5 animals were left untreated. G2: 5 animals, their right buccal pouches were painted with $0.5 \% 7,12$ dimethylbenz [a] anthracene (DMBA) 3 times a week for 8 weeks. G3: 5 animals were received GSH one week before, as well as during the application of DMPA for 8 weeks. G4: 5 animals were received Se one week before, as well as during the application of DMPA for 8 weeks. G5: 5 animals were received GSH and Se one week before, as well as during the application of DMPA for 8 weeks. After termination of the experiment, all animals were sacrificed, and the buccal mucosa was excised and preparation in order to be examined histologically and immunohistochemically, then statistical analysis based on these examinations was done
\end{abstract}

Results: Regard to expression of $\mathrm{Bcl}-2, \mathrm{G} 2$ had recorded the highest mean area percentage (44.25\%), while G1 had recorded the lowest mean area percentage $(6.73 \%)$ and there was high significant difference between G1,G2, G3, G4 and G5 where P value was $<0.001$, while in regard to expression of Bak, G1 had recorded the highest mean area percentage (41.02\%), while G2 had recorded the lowest mean area percentage $(19.79 \%)$ and there was significant difference between G1, G2, G3, G4 and G5 where P value was 0.019 .

Conclusion: GSH and Se are considered as promising chemotherapeutic agents in prevention of induced HBP epithelial dysplasia and proved important role in apoptosis and proliferation throughout the process of carcinogenesis.

KEYWORDS: HBP carcinogensis, GSH, Se, Bcl2, Bak.

* Lecturer of Oral Pathology - Faculty of Dentistry - Al-Azhar University.

** Assistant Professor of Oral Biology - Faculty of Dentistry - Al-Azhar University. 


\section{INTRODUCTION}

Cancer is a multistep disease process mediated by a diversity of endogenous and environmental stimuli. Reactive oxygen species (ROS) and other free radicals are postulated to be involved in neoplastic transformation. ROS are generated endogenously and exogenously as a by-product of normal respiration and as a function of biochemical reactions using oxygen ${ }^{[1]}$. ROS at high levels are toxic to the cell, but at low levels, ROS have physiological functions, including activation and modulation of signal transduction pathways, modulation of activities of redoxsensitive transcription factors, and regulation of mitochondrial enzyme activities ${ }^{[2]}$. To protect the cells and organ systems of the body against ROS, humans have evolved a highly sophisticated and complex antioxidant protection system. It involves a variety of components, that function interactively and synergistically to neutralize free radicals. These include antioxidants like Glutathione (GSH) ceruloplasmin, lipoic acid, ascorbic acid (Vitamin C), tocopherols and tocotrienols (Vitamin E), carotenoids ${ }^{[3]}$. GSH is a tripeptide produced naturally in the body, with an intracellular concentration of $1-10 \mathrm{mM}$ in mammalian cells. It is the most abundant non-protein molecule in the cell and has a number of physiological roles. GSH is synthesized intracellularly from its constituent amino acids: glutamic acid, cysteine and glycine via two sequential ATP-consuming steps, which are catalysed by glutamylcysteine synthetase (GCS) and GSH synthase ${ }^{[4]}$. Oxidation of the reduced GSH to form oxidized glutathione (GSSG) is carried out either by direct interaction with free radicals or, more often, when GSH acts as a cofactor for antioxidant enzymes such as GSH peroxidases during the reduction of $\mathrm{H} 2 \mathrm{O} 2$ and phospholipid hydroperoxide GSH peroxidases ${ }^{[5]}$. When intracellular GSH levels are low, the cells are more vulnerable to ROS attacks. Increased ROS might activate different intracellular oncogenic pathways or mutate a tumor suppressor gene pathway, which will activate a tumorigenesis process ${ }^{[6]}$. Because the increase of ROS in cancer cells may be part of the initiation and progression of cancer, such intrinsic oxidative stress is often viewed as an adverse event. However, as excessive levels of ROS stress can also be toxic to the cancer cells and cells are likely to be more vulnerable to damage by further ROS induced by exogenous drugs and make them more responsive to ROS producing cancer treatments. Therefore, changing ROS levels by GSH modulation is a way to selectively kill cancer cells without causing significant toxicity to normal cells ${ }^{[7]}$. On the other hand, GSH is important in the detoxification of carcinogens, its elevated state in many types of tumors may also increase resistance or alters the cytotoxicity of many chemotherapy drugs or radiation ${ }^{[8]}$. Selenium (Se) is an oligoelement with essential biological functions and belongs to the most extensively studied chemopreventive compounds ${ }^{[9]}$. An adequate Se intake has been estimated at $50 \mu \mathrm{g} / \mathrm{day}$ with toxic levels being estimated to occur with intakes of the order of $350-700 \mu \mathrm{g} /$ day ${ }^{[10]}$. Se is important for many cellular processes, because it is a component of several selenoproteins with preventive function of some forms of cancer ${ }^{[11]}$. Se supplementation with low doses seems to be beneficial not only for cancer prevention, but it can positively influence many other functions in an organism by reducing inflammations, heart diseases and regulating the blood pressure ${ }^{[12]}$. Effectiveness of Se compounds as chemopreventive agents in vivo is correlated with their abilities to effect the regulation of the cell cycle, to stimulate apoptosis and to inhibit tumor cell migration and invasion in vitro ${ }^{[13]}$. Besides, it is widely recognized that the effectiveness of Se compounds as chemopreventive and anticancer agents is correlated with their chemical form and doses. In the present study, we tested the hypothesis that oral tumor modulation by GSH and Se may be mediated, in part, through the changes in the activities and expression of apoptotic protein. 


\section{MATERIAL AND METHODS}

Twenty five golden Syrian male hamsters five weeks old, weighting 80-120gs were obtained from the animal house, Cairo University (Cairo, Egypt). The experimental animals were housed in standard cages with sawdust bedding under controlled environmental conditions of humidity (30-40\%), temperature $\left(20 \pm 2^{\circ} \mathrm{C}\right)$, and light $(12-$ h light/12-h dark). All experimental animals were supplied with standard diet and water ad libitum. The hamsters were used as model for oral squamous cell carcinoma (OSCC) induction utilizing 7, 12 dimethylbenz[a]anthracene $(\mathrm{DMBA})^{1} \quad(0.5 \%$ in paraffin oil) as chemical carcinogen also GSH and Selenium were used as chemoprevention agent. The experimental animals were divided into three groups. G1 (negative control): 5 hamsters not treated and served as negative controls. G2 (DMBA paintinghamster buccal pouch (HBP) group): 5 hamsters were included in this group, the right HPPs of these hamsters were painted with 0.5 DMBA in paraffin using a number 4 camel hair brush three times a week for 8 weeks. G3 (GSH chemoprevention group) ${ }^{1}$ : 5 hamsters were included in this group were received 84 ppm GSH ${ }^{[14]}$ given by the oral route using a specific vehicle one week before, as well as during the application of DMBA on alternative days for 8 weeks. G4 (Selenium chemoprevention group): 5 hamsters were included in this group were received Se $10 \mathrm{mg} / \mathrm{kg}[15]^{1}$ given by the oral route using a specific vehicle one week before, as well as during the application of DMBA on alternative days for 8 weeks. G5 (GSH and Selenium chemoprevention group): 5 hamsters were included in this group were received 84 ppm GSH and $\mathrm{Se}^{[14]}$ given by the oral route using a specific vehicle one week before, as well as during the application of DMBA on alternative days for 8 weeks. After termination of the experiment and recording all gross observations and alterations that may happened throughout the experiment, the animals were sacrificed by cervical dislocation, the cheek pouches were excised and fixed in $10 \%$ neutral buffered formalin, routinely processed and embedded in paraffin blocks for preparation in order to be examined histologically and immunohistochemically and then statistical analysis based on these examinations was done. For histological examination: The specimens were dehydrated in an ascending ethanol series, embedded in paraffin wax to form paraffin blocks. Tissue sections using rotary microtome of $4 \mu \mathrm{m}$ thickness were cut, mounted on glass slides and stained with hematoxylin and eosin stain (H\&E) for light microscopic examination.Forimmunohistochemical (IHC) examination: Other tissue sections were cut at $4 \mu \mathrm{m}$ and put on positive charged slides for the application of standard labeled streptavidin- biotin method to apply each antibody used separately (Bcl2 and Bak antibodies) $)^{2}$. Each section was carried into two similar sections one for Bax and one for Bcl-2.The sections were deparaffinized in xylene and rehydrated through graded ethanol $(100 \%, 95$ $\%$ and $70 \%$ ) each run for 5 minutes. Slides were washed in distilled water then in phosphate buffered saline (PBS), each for 5 minutes. Endogenous peroxidase activity was blocked using $3 \%$ solution of hydrogen peroxide (H2O2) in methanol for 30 minutes at room temperature. Slides were then washed in PBS. Slides were then immersed in plastic jars containing $200 \mathrm{ml}$ of $10 \mathrm{M}$ citrate buffer (pH 6) (ready to use from DAKO). The jars were put in microwave at maximum power at $100^{\circ} \mathrm{C}$ for 3 intervals, each one 5 minutes. Slides were left at room temperature to coal gradually. Slides were then washed in distilled water followed by PBS for 5 minutes. Tissue sections were received one or two drops of the primary antibodies (Bak or Bcl2) in a dilution of 1:100 and incubated in a humid chamber at room temperature overnight. Slides were then washed in distilled water, followed by PBS for 5 minutes. Biotinylated secondary antibody was added and incubated at room temperature for 30 minutes. Tissue sections were then washed in PBS for 5 minutes. One or two drops of peroxidase- 
labeled streptavidin were applied for 30 minutes at room temperature then washed in PBS. The tissue sections were received $\mathrm{DAB}$ for 2-4 minutes to develop color, followed by putting in distilled water. Tissue sections were counterstained using Mayer's hematoxylin for one minute and then washed in tap water.

The slides were placed in two changes of $95 \%$ alcohol followed by two changes of absolute alcohol, each for 3 minutes then mounted with DPX and covered with plastic covers in order to be examined. The immunostained sections were examined using light microscope to assess the prevalence of positive cases and the localization of immmunostaining within the tissues. In addition, image analysis computer system $^{3}$ was used to assess area percentage of positive cells of the immunostaining.

This was done in the Oral and Dental Pathology Department - Faculty of Dental Medicine - BoysCairo - Al-Azhar University. The degree of positive staining for each antibody was evaluated by a wellestablished semi-quantitative scoring on a scale range from negative to strong positive staining as follow: Strong staining (more than 50\% stained), moderate staining (between 25 and 50\% stained), weak staining (between 5 and $25 \%$ stained), and negative (less than $5 \%$ stained) ${ }^{[16]}$.

Statistical presentation and analysis of the present study was conducted, using the mean, standard deviation, ANOVA and Tukey's test by SPSS V20 ${ }^{4}$. Significant level: Non Significant $>0.05$ Significant $<0.05^{*}$ High Significant $<0.001 *$

1: Sigma-aldrich company

2: Thermo Fisher Scientific, USA

3: Software Leica Quin 500, Wetzlar, Germany

4: IBM SPSS Statistics, London, UK

\section{RESULTS}

The gross observation results: HBP mucosae of G1 were pink in color with smooth surface and no observable abnormalities (Fig. 1, A ), in G2, HBP mucosa showed multiple small nodules, large area of ulceration and bleeding (Fig. 1, B), in G3 HBP mucosae showed multiple small nodules with ulceration and bleeding (Fig. 1, C), in G4 HBP mucosae showed small nodules with ulceration and without bleeding (Fig. 1, D), in G5 HBP mucosae showed few small nodules without bleeding or ulceration (Fig. 1, E). Histopathological and IHC results: In G1, histological sections, using $\mathrm{H} \& \mathrm{E}$ stain, revealed normal HBP mucosae, intact, and continuous epithelium composed of thin keratinized stratified squamous epithelium.

Subepithelial connective tissue (C.T), muscular layer and areolar layer were seen (Fig. 2, A). The IHC staining using Bcl-2 showed weak $(6.73 \pm 4.25$ $\%)$ positive expression which which limited to basal and suprabasal layers (Fig. 2, B). while Bak expression exhibited moderate $(41.02 \pm 10.08 \%)$ positive expression which present throughout the epithelial layers (Fig. 2, C). Histological sections, using H\&E stain of G2 showed $100 \%$ incidence of epithelial dysplasia, the overlying epithelium has obvious, hyperplasia, hyperkeratosis and severe included the above parameters extending beyond one half of the epithelial thickness but not affecting the entirety of the epithelium: additional features included loss of adhesion, hyperchromatism and abnormal mitosis with intact basement membrane (Fig. 2, D). IHC staining using Bcl-2 expression exhibited moderate $(44.2 \pm 14.24 \%)$ positive cytoplasmic expression throughout the dysplastic epithelial layers (Fig. 2, E), also Bak of G2 showed weak $(19.79 \pm 7.12 \%)$ positive cytoplasmic expression throughout the dysplastic epithelial layers (Fig. 2, F). Histological sections, using H\&E stain of G3 reduced epithelial dysplasia incidence and severity of three premalignant lesions as 

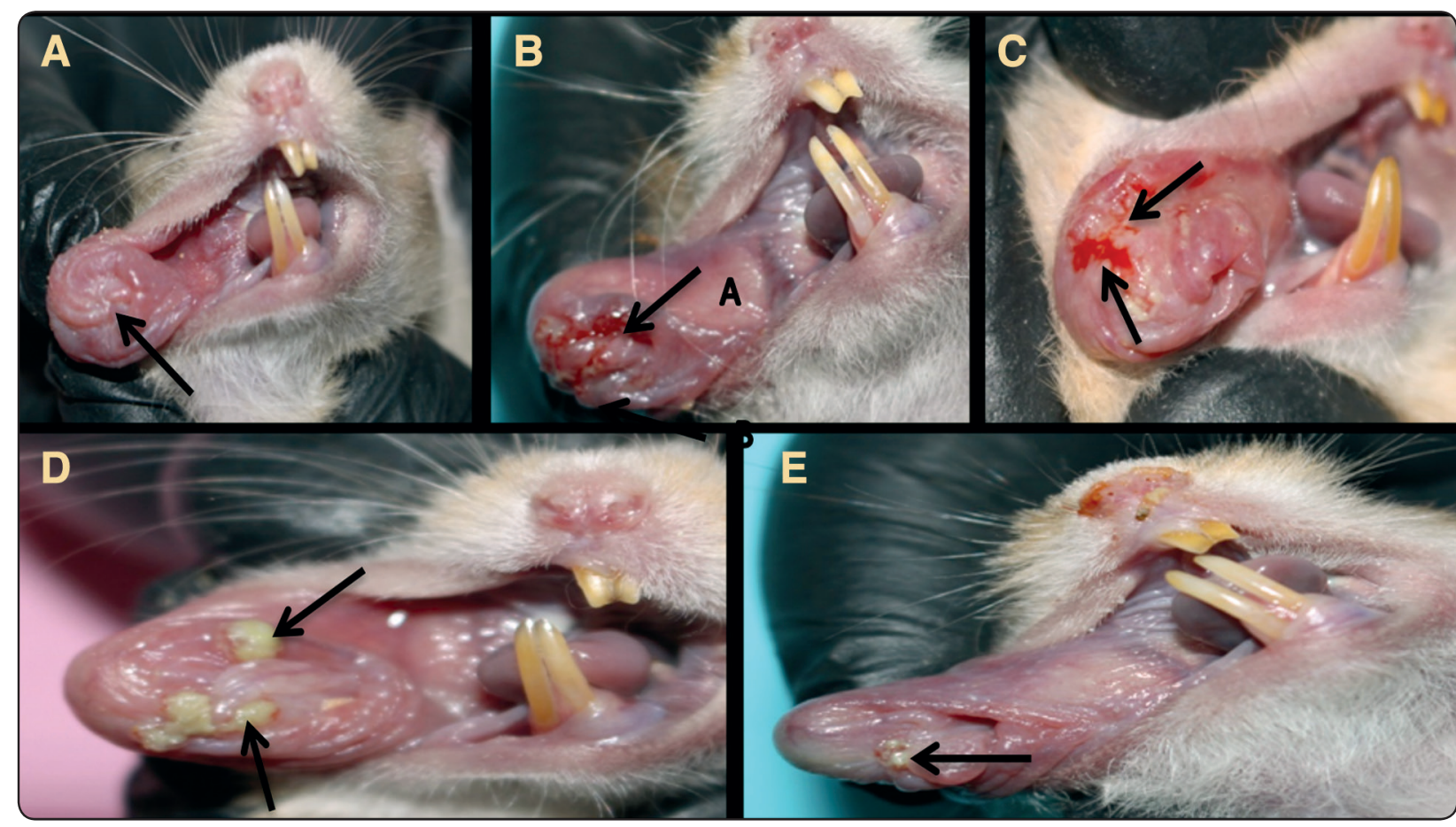

Fig. (1) HBP of GI showing normal buccal pouch mucosa which appeared pink in color with smooth surface (arrow) (A). HBP of G2, showing multiple small nodules, (arrow A), area of ulceration and bleeding (arrow B) (B). HBP of G3 showing multiple small nodules with ulceration and bleeding (arrows) (C). HBP of G4 showing multiple small nodules with ulceration and without bleeding (arrows) (D). HBP of G5 showing few small nodules without bleeding or ulceration (arrow) (E).

compared to G2, (2 animals exhibited moderate epithelial dysplasia and 1 animals exhibited severe epithelial dysplasia in most epithelial layers in multiple areas including: loss of adhesion and abnormal mitosis with intact basement membrane) (Fig. 2, G)., while two specimen appeared normal almost the same as G1, IHC staining using Bcl2 expression exhibited moderate $(28.58 \pm 9.15 \%)$ positive cytoplasmic expression throughout the dysplastic epithelial layers (Fig. 2, H)., Bak showed moderate $(27.57 \pm 5.91 \%)$ positive cytoplasmic expression throughout the dysplastic epithelial layers (Fig. 2, I).

Histological sections, using H\&E stain of G4 reduced epithelial dysplasia incidence and severity of two premalignant lesions as compared to G2, exhibited moderate epithelial dysplasia (Fig. 3, A), while three specimen appeared normal almost the same as G1, IHC staining using Bcl-2 expression exhibited weak $(20.88 \pm 5.06 \%)$ positive cytoplasmic expression throughout the dysplastic epithelial layers (Fig. 3, B), Bak showed moderate $(32.17 \pm 11.67 \%)$ positive cytoplasmic expression throughout the dysplastic epithelial layers (Fig. 3, C). Histological sections, using H\&E stain of G5 reduced epithelial dysplasia incidence and severity of one premalignant lesions as compared to G2, exhibited mild epithelial dysplasia (Fig. 3, D), while four specimen appeared normal almost the same as G1, IHC staining using Bcl-2 expression exhibited weak (12.36 $\pm 5.61 \%)$ positive cytoplasmic expression throughout the dysplastic epithelial layers (Fig. 3, E), Bak showed moderate $(37.22 \pm 15.84 \%)$ positive cytoplasmic expression throughout the dysplastic epithelial layers (Fig. 3, F). Statistical analysis results were revealed that, in regard to expression of Bcl-2 at 8 weeks, G2 had recorded the highest mean area percentage $(44.25 \%)$, while G1 had recorded the 
lowest mean area percentage (6.73\%) and there was high significant difference between G1,G2, G3, G4 and G5 where P value was $<0.001$, also there was high significant difference between the following groups (G1 and G2), ( (G4 and G2) and (G5 and $\mathrm{G} 2)$, respectively where $\mathrm{P}$ value was $(<0.001)$ and there was significant difference between G3 and G2 where P value was (0.028) (Table 1, Chart 1). While in regard to expression of Bak at 8 weeks, G1 had recorded the highest mean area percentage
(41.02\%), while G2 had recorded the lowest mean area percentage $(19.79 \%)$ and there was significant difference between G1, G2, G3, G4 and G5 where $\mathrm{P}$ value was 0.019 ,. also there was significant difference between $\mathrm{G} 1$ and $\mathrm{G} 2$ where P value was (0.016) and there was no significant difference between the following groups (G3 and G2), ( (G4 and G2) and (G5 and G2), where P value was (0.718, $0.294,0.065$ ) respectively (Table 2 , Chart 2).

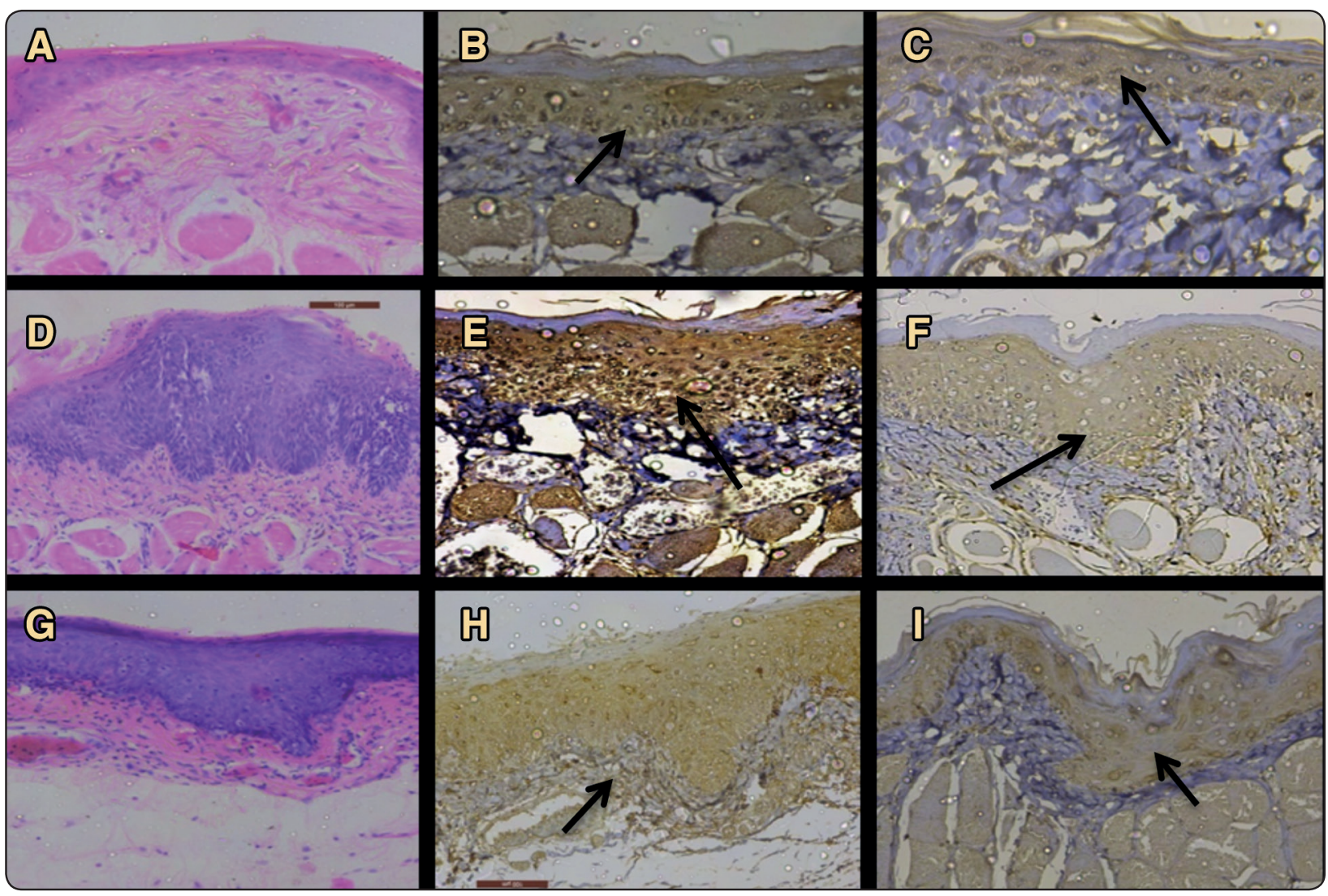

Fig. (2) H\&E stain of G1 showing: thin keratinized stratified squamous epithelium with flattened rete ridges and sub epithelial connective tissue (A). IHC expression of Bcl-2 showing positive cytoplasmic expression which limited to basal and suprabasal layers (arrow) (B). IHC expression of Bak showing positive cytoplasmic expression throughout the epithelial layers (arrow) (C). H\&E stain of G2 showing sever epithelial dysplasia (D). IHC expression of Bcl-2 showing positive cytoplasmic expression throughout the dysplastic epithelial layers (arrow) (E). IHC expression of Bak showing positive cytoplasmic expression throughout the dysplastic epithelial layers (arrow) (F). H\&E stain of G3 showing: showing moderate epithelial dysplasia (G). IHC expression of Bcl-2 showing positive cytoplasmic expression throughout the dysplastic epithelial layers (arrow) (H). IHC expression of Bak showing positive cytoplasmic expression throughout the dysplastic epithelial layers (arrow). (H\&E stain x 100) (Bcl-2 stain x 200) (Bak stain x 200) 


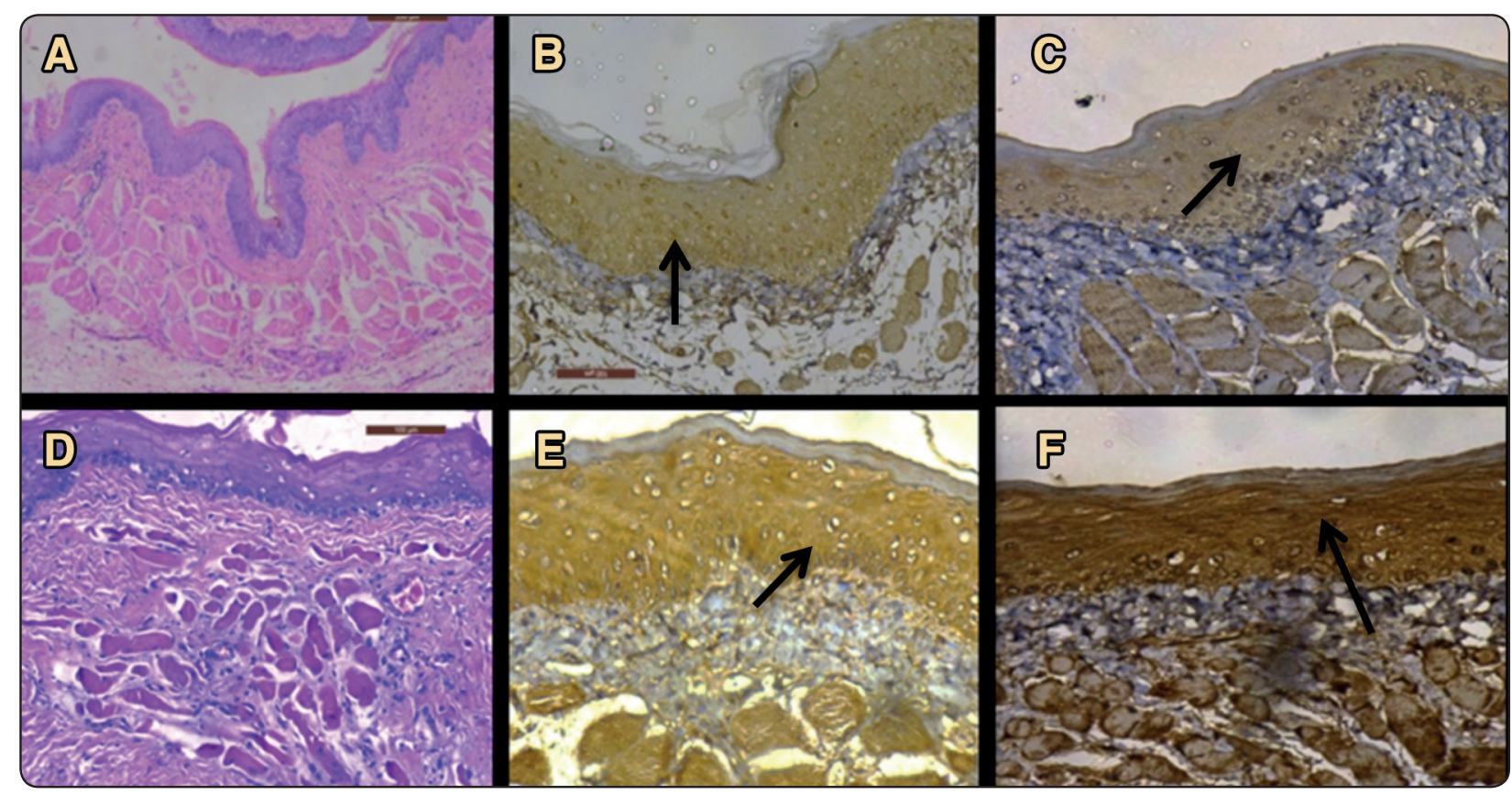

Fig. (3) H\&E stain of G4 showing moderate epithelial dysplasia (A). IHC expression of Bcl-2 showing positive cytoplasmic expression throughout the dysplastic epithelial layers (arrow) (B). IHC expression of Bak showing positive cytoplasmic expression throughout the dysplastic epithelial layers (arrow) (C). H\&E stain of G5 showing: showing mild epithelial dysplasia (D). IHC expression of Bcl-2 showing positive cytoplasmic expression throughout the dysplastic epithelial layers (arrow) (E). IHC expression of Bak showing positive cytoplasmic expression throughout the dysplastic epithelial layers (arrow) (F). (H\&E stain x 100) (Bcl-2 stain x 200) (Bak stain x 200)

TABLE (1) Mean, standard deviation (SD), P-values and results of comparison between expression of Bcl-2 all groups.

\begin{tabular}{|c|c|c|c|c|c|c|c|c|}
\hline \multirow{2}{*}{ Groups } & \multicolumn{6}{|c|}{ Bcl2 } & \multicolumn{2}{|c|}{ ANOVA } \\
\hline & \multicolumn{3}{|c|}{ Range } & Mean & \pm & SD & $\mathrm{f}$ & P-value \\
\hline G1 & 2.3 & - & 14.7 & 6.73 & \pm & 4.25 & \multirow{5}{*}{17.924} & \multirow{5}{*}{$<0.001 * *$} \\
\hline G2 & 24.1 & - & 66.6 & 44.25 & \pm & 14.24 & & \\
\hline G3 & 16.0 & - & 39.9 & 28.58 & \pm & 9.15 & & \\
\hline G4 & 12.6 & - & 25.3 & 20.88 & \pm & 5.06 & & \\
\hline G5 & 5.4 & - & 20.2 & 12.36 & \pm & 5.61 & & \\
\hline
\end{tabular}

Tukey's test

\begin{tabular}{|c|c|c|c|c|}
\hline & G1 & G2 & G3 & G4 \\
\hline G2 & $<0.001 * *$ & & & \\
\hline G3 & $<0.001 * *$ & $0.028 *$ & 0.530 & \\
\hline G4 & 0.056 & $<0.001 * *$ & $0.022^{*}$ & 0.432 \\
\hline G5 & 0.781 & $<0.001 * *$ & & \\
\hline
\end{tabular}


TABLE (2) Mean, standard deviation (SD), P-values and results of comparison between expression of Bcl-2 all groups.

\begin{tabular}{|c|c|c|c|c|c|c|c|c|}
\hline \multirow{2}{*}{ Groups } & \multicolumn{6}{|c|}{ Bak } & \multicolumn{2}{|c|}{ ANOVA } \\
\hline & \multicolumn{3}{|c|}{ Range } & Mean & \pm & SD & $\mathrm{f}$ & P-value \\
\hline G1 & 30.7 & - & 60.3 & 41.02 & \pm & 10.08 & \multirow{5}{*}{3.609} & \multirow{5}{*}{$0.019 *$} \\
\hline G2 & 11.5 & - & 32.6 & 19.79 & \pm & 7.12 & & \\
\hline G3 & 21.0 & - & 37.1 & 27.57 & \pm & 5.91 & & \\
\hline G4 & 22.2 & - & 54.3 & 32.17 & \pm & 11.67 & & \\
\hline G5 & 18.6 & - & 66.2 & 37.22 & \pm & 15.84 & & \\
\hline
\end{tabular}

\section{Tukey's test}

\begin{tabular}{|c|c|c|c|c|}
\hline & G1 & G2 & G3 & G4 \\
\hline G2 & $0.016^{*}$ & & & \\
\hline G3 & 0.223 & 0.718 & & \\
\hline G4 & 0.615 & 0.294 & 0.944 & 0.923 \\
\hline
\end{tabular}

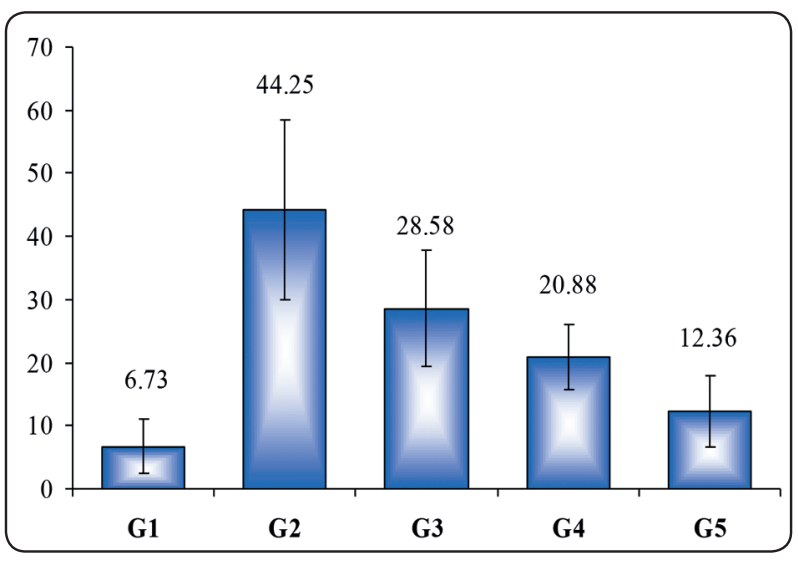

Chart (1): Bar chart representing mean area \% results of Bcl-2 expressions in all groups.

\section{DISCUSSION}

In the present study, the results of the effect of GSH and Se as a new chemopreventive modality in experimentally induced HBP carcinogenesis revealed variable alterations. Despite the existence of anatomic and histologic variations between hamster pouch mucosa and human buccal tissue, the DMBA-treated hamster cheek pouch model is able to produce premalignant changes and carcinomas

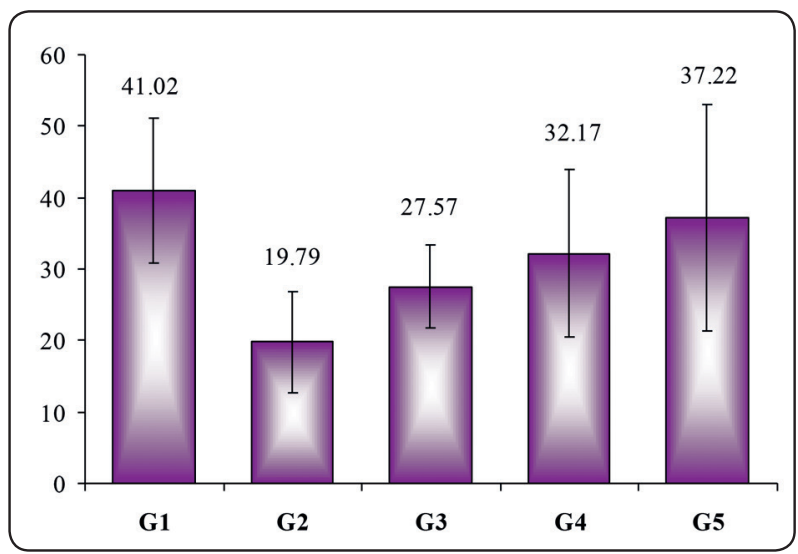

Chart (2): Bar chart representing mean area \% results of Bak expressions in all groups.

that are similar to the development of disease in human oral mucosa [17]. The gross observation in G1 (control: untreated animals) showed no observable gross changes, HBP mucosa appeared normal, the weak positive cytoplasmic expression of Bcl-2, in the basal and suprabasal epithelial layers, is in agreement with those of other investigators [18 - 20]. The moderate cytoplasmic expression of Bak, in the superficial epithelial layer, is in 
agreement with those of other investigators [21,22]. This result may be due to that; Bcl-2 participates in the control of the terminal differentiation of keratinocytes by protecting their stem cells from apoptosis. In the present study epithelial dysplasia was observed After DMBA painting for 8 weeks (G2). The activities of $\mathrm{Bcl} 2$ were increased with the progression of HBP carcinogenesis. The moderate expressions were observed in sever epithelium dysplasia while the activities of Bak were decreased with the progression of HBP carcinogenesis, the weak expressions were observed in sever epithelium dysplasia and there was high significant difference between $(\mathrm{G} 1$ and $\mathrm{G} 2)$, where P value was $(<0.001)$ regarding to $\mathrm{Bcl} 2$ expression and between $(\mathrm{G} 1$ and $\mathrm{G} 2)$ where $\mathrm{P}$ value was $(<0.05)$ regarding to Bak expression. The trend observed in the present study was consistent with the results of previous studies [23, 24,]. DMBA carcinogen disrupts the prooxidant antioxidant balance, which finally leads to antioxidants depletion in cells ( $\mathrm{Li}$ et al., 2002) [25]. Krishnaveni and Mirunalini (2012) [26] had reported decreased levels of lipid peroxidation (LPO) and antioxidants in tumor tissues, which could be due to prolonged tumour cells proliferation. Antioxidants such as GSH, ceruloplasmin, alpha tocopherol etc are capable of stabilizing, or deactivating, free radicals before they attack normal cells and prevent their conversion to cancer cells. Gross observation of G3 model revealed decrease in distribution and size of the nodules, ulcerative and bleeding areas compared to G2, also in G3 reduced epithelial dysplasia incidence, three specimen with moderate to severe dysplastic while two specimen appeared normal almost the same as G1. There was significant difference between (G3 and G2), where $\mathrm{P}$ value was (0.028) regarding to $\mathrm{Bcl} 2$ expression and there was no significant difference between (G3 and G1) where $P$ value was (0.223) regarding to Bak expression. The present study suggested that the chemopreventive effect of GSH was accomplished by inhibiting tumor cell proliferation and inducing tumor cell apoptosis, Dormandy had proposed a close relationship between free radical activity and malignancy [27]. It must be noted that, Beutler and Kelly's method measures the total thiols including GSH. However it has been recognized that the levels of total thiols fairly well correlate with levels of GSH [28]. GSH is a major intracellular antioxidant and hence plays a major role in cancer prevention [29]. GSH acts as first line of defense against oxidative stress [30]. Few studies have shown a reduced occurrence of polycyclic aromatic hydrocarbon induced oral cancer in experimental animals fed with diet rich in GSH [31, 32]. GSH detoxifies carcinogens and also regulates immune system by mitogenic response and lymphocytic proliferation [33]. Several serum studies have revealed decrease in the levels of plasma GSH levels in oral cancer [34]. In one Indian study the levels of plasma GSH consistently reduced in the advanced stages of oral cancer when compared to initial stages [35]. The protective effect of GSH was attributed to its ability to stabilize the pollutants by scavenging the free radicals and thereby blocking LPO development [36]. In the present study, gross observation of G4 model revealed decrease in distribution and size of the nodules, ulcerative and bleeding areas compared to $\mathrm{G} 2$, and $\mathrm{G} 3$ also in $\mathrm{G} 4$ reduced epithelial dysplasia incidence, two specimen with moderate dysplastic while three specimen appeared normal almost the same as G1. There was high significant difference between $(\mathrm{G} 4$ and $\mathrm{G} 2)$, where P value was $(<0.001)$ regarding to $\mathrm{Bcl} 2$ expression and there was no significant difference between (G4 and G1) where $\mathrm{P}$ value was (0.615) regarding to Bak expression. Se play important role in prevention oral squamous cell carcinoma. Human epidemiological studies conducted over this period of time examined the relationship between dietary intake of Se and total cancer risk, and have been somewhat controversial [37]. In another study the effect of low Se concentrations in serum on the incidence of different cancers and on total death were followed. 
Significant inverse association between base-line serum Se and death from esophageal and gastric [38] have been found. Several proposed mechanisms to explain the effect of Se on cell cycle and apoptosis and it has been well recognized that Se plays a key role in these processes but mechanisms for Se action are very complex and not fully understood. They involve protein kinases signaling, activation of caspases, p53 phosphorylation and ROS generation [39]. These results suggest that Se supplementation may have some protective effects against some type(s) of cancer in populations where average dietary Se levels are low. In the present study, gross observation of G5 model revealed decrease in distribution and size of the nodules, ulcerative and bleeding areas compared to G2, G3, G4 also in $\mathrm{G} 5$ reduced epithelial dysplasia incidence, one specimen with mild dysplastic while four specimen appeared normal almost the same as G1, also there was high significant difference between (G5 and $\mathrm{G} 2)$, where $\mathrm{P}$ value was $(<0.001)$ regarding to $\mathrm{Bcl} 2$ expression and there was no significant difference between (G5 and G1) where P value was (0.972) regarding to Bak expression. In the present study, the combination GSH and Se (selenodiglutathione) hold great promise as chemopreventive agents oral carcinogenesis These selenodiglutathione agents were found to be superior to historically used Se alone. There is evidence in support of the concept that the reductive metabolism of selenite by GSH, which leads to the formation of the primary metabolite, selenodiglutathione, is a prerequisite for its antiproliferative effect [40]. Hasegawa et al. [40] identified Se-GSH selenyl sulfide as the Se-containing metabolite in the small intestine of mice treated with Se. Therefore, Rao, et al (2001) (14) hypothesize that GSH conjugates are putative metabolites that will be more effective than their parent organoselenium compounds. On the basis of these studies, Se-SG is a primary metabolite of Se mediating the chemopreventive activity and is a better inhibitor of colon carcinogenesis than other organoselenium compounds thus far evaluated for their efficacy [14]. Rao, et al (2001) (14) demonstrates that dietary administration of a putative metabolite of $\mathrm{p}$-XSC namely, $\mathrm{p}-\mathrm{XSe}-\mathrm{SG}$, during the post-initiation stage of AOM-induced carcinogenesis in rats significantly suppresses colon adenocarcinoma formation in the colon in a dosedependent manner. Furthermore, this new agent possesses very low-toxicity, COX-2-inhibitory activity and relatively higher chemopreventive index compared with previously known organoselenium compounds tested in the experimental colon cancer using similar experimental design and protocols.

\section{CONCLUSION}

This study prove evidence that the diet-derived GSH and Se, exert anticancer effects mediated through induction of apoptosis and suppressing cell invasion also combination between GSH and Se is considered as a promising chemotherapeutic agents in prevention of induced HBP epithelial dysplasia. In addition, the use of GSH and Se derivatives was found to be safe without any indicated side effects.

\section{REFERENCES}

1. Chance B, Sies H, Boveris A. Hydroperoxide metabolism in mammalian organs. Physiol Rev. 1979; 59: 527-605.

2. Nulton-persson AC, Szweda LI. Modulation of mitochondrial function by hydrogen peroxide. J Biol Chem 2001; 276: 23357-61.

3. Sawair FA, Irwin CR, Gordon DJ, Leonard AG, Stephenson M, Napier SS. Invasive front grading: reliability and usefulness in management of oral squamous cell carcinoma. J Oral Pathol Med 2003; 32: 1-9.

4. Rahman I. Regulation of nuclear factor-kappa B, activator protein-1, and glutathione levels by tumor necrosis factoralpha mand dexamethasone in alveolar epithelial cells. Biochem Pharmacol 2000; 60: 1041-49.

5. Holmgren A. Thioredoxin and glutaredoxin systems. J Biol Chem 1989; 264: 13963-66.

6. Komatsu D, Kato M, Nakayama J, Miyagawa S, Kamata T. NADPH oxidase 1 plays a critical mediating role in 
oncogenic Ras-induced vascular endothelial growth factor expression. Oncogene 2008; 27: 4724-32.

7. Trachootham D, Alexandre J, Huang P. Targeting cancer cells by ROS-mediated mechanisms: a radical therapeutic approach. Nat Rev Drug Discov 2009; 8: 579-91.

8. Ganesaratnam K, Balendiran, Dabur R, Fraser D. The role of glutathione in cancer. Cell Biochem. Function 2004; 22: 343-52.

9. Thomson C.D. Assessment of requirements for selenium and adequacy of selenium status: A review. Eur. J. Clin. Nutr. 2004; 58: 391-402.

10. Council, N.R. Dietary Reference Intakes for Vitamin C, Vitamin E, Selenium, and Carotenoids; The National Academies Press: Whasington, DC, USA, 2000.

11. Lu, J., Holmgren A. Selenoproteins. J. Biol. Chem. 2009; 284, 723-27.

12. Brozmanova J, Manikova D, Vlckova V, Chovanec M. Selenium: A double-edged sword for defense and offence in cancer. Arch. Toxicol 2010; 84: 919-38.

13. Zeng H, Combs, G.F., Jr. Selenium as an anticancer nutrient: Roles in cell proliferation and tumor cell invasion. J. Nutr. Biochem 2008; 19: 1-7.

14. Rao CV1, Wang CQ, Simi B, Rodriguez JG, Cooma I, ElBayoumy K, et al. Chemoprevention of Colon Cancer by a Glutathione Conjugate of 1,4-Phenylenebis(methylene) selenocyanate, a Novel Organoselenium Compound with Low Toxicity1. Cancer Res 2001; 61:3647-52.

15. Reddy B S, Rivenson A, El-Bayoumy K, Upadhyaya P, Pittman B, Rao CV. chemoprevention of colon cancer by Organoselenium compounds and impact of high- or lowfat diets. J. Natl. Cancer Inst 1997; 89: 506-12.

16. Negi A, Puri A, Gupta R, Nangia R, Sachdeva A, Mittal M. Comparison of immunohistochemical expression of antiapoptotic protein survivin in normal oral mucosa, oral leukoplakia, and oral squamous cell carcinoma. Patholog Res Int 2015; 2015:1-6.

17. Morris A.L. Factors influencing experimental carcinogenesis in the hamster cheek pouch. J. Dent Res 1961; 40: $3-15$.

18. Kathiresan S, Mariadoss A, Muthusamy R, Kathiresan S. Protective effects of [6]-shogaol on histological and immunohistochemical gene expression in DMBA induced hamster buccal pouch carcinogenesis. J Asian Pacific Cancer Prev 2013; 14: 3123-29.
19. Balakrishnan S, Manoharan S, Alias L, Nirmal M. Effect of curcumin and ferulic acid on modulation of expression pattern of p53 and bcl-2 proteins in 7, 12-dimethylbenz anthracene-induced hamster buccal pouch carcinogenesis. J Indian Biochem Biophys 2010;47:7-12.

20. Rajasekaran D, Manoharan S, Silvan S, Vasudevana K, Baskaran N, Palanimuthu D. Proapoptotic, anti-cell proliferative, anti-inflammatory and antiangiogenic potential of carnosic acid during 7, 12 dimethylbenz [a] anthracene-induced hamster buccal pouch carcinogenesis. J Afr Tradit Complement Altern Med 2013;10: 102-12 .

21. Manoharan S, Palanimuthu D, Baskaran N, Silvan S. Modulating effect of lupeol on the expression pattern of apoptotic markers in 7, 12-dimethylbenz (a) anthracene induced oral carcinogenesis. J Asian Pac Cancer Prev 2012; 13: 5753-57.

22. Zhang W, Yin G, Dai J, Sun YU, Hoffman RM, Yang Z, et al. Chemoprevention by Quercetin of Oral Squamous Cell Carcinoma by Suppression of the NF-kb Signaling Pathway in DMBA-treated Hamsters. Anticancer Res 2017; 37: 4041-49.

23. Yang P, Sun Z, Chan D, Cartwright CA, Vijjeswarapu M, Ding J, etal. Zyflamend_ reduces LTB4 formation and prevents oral carcinogenesis in a 7,12- dimethylbenz[a] anthracene (DMBA)-induced hamster cheek pouch model. Carcinogenesis 2008; 29: 2182-89.

24. Kathiresan S, Govindhan A. [6]-Shogaol, a Novel Chemopreventor in 7,12- Dimethylbenz[a]anthraceneinduced Hamster Buccal Pouch Carcinogenesis. Phytother Res 2016; 30:646-53.

25. Li N, Chen X, Liao J, Yang G. Inhibition of 7,12 dimethylbenz[a]anthracene (DMBA) induced oral carcinogenesis in hamsters by tea and curcumin. Carcinogenesis 2002; 23: 1307-13.

26. Krishnaveni M, Mirunalini S. Chemopreventive efficacy of Phyllanthus emblica L. (amla) fruit extract on 7,12dimethylbenz(a)anthracene induced oral carcinogenesisA dose-response study. Environ Toxicol Pharmacol 2012; 34: 801-10.

27. Dormandy TL. An approach to free radicals. Lancet 1983; 2: 1010-14.

28. Jaswal S, Mehta HC, Sood AK, Kaur J. Antioxidant status in rhuematoid arthritis and role of antioxidant therapy. Clin Chim Acta 2003; 338: 123-29. 
29. Locigno R, Castronovo V. Reduced glutathione system: role in cancerdevelopment, prevention and treatment (review). Int J Oncol 2001; 19: 221-36.

30. Sies H. Glutathione and its role in cellular functions. Free Radic Biol Med 1999; 27: 916-21.

31. Trickler D, Shklar G, Schwartz J. Inhibition of oral carcinogenesis by glutathione. Nutr Cancer 1993; 20: 139- 44.

32. Schwartz JL, Shklar G. Glutathione inhibits experimental oral carcinogenesis, p53 expression, and angiogenesis. Nutr Cancer 1996; 26: 229-36.

33. Wu G, Fang YZ, Yang S, Lupton JR, Turner ND. Glutathione metabolism and its implications for health. J Nutr 2004; 134: 489-92.

34. Richie JP, Kleinman W, Marina P, Abraham P, Wynder EL, Muscat JE. Blood Iron, Glutathione, and Micronutrient Levels and the Risk of Oral Cancer. Nutr Cancer 2008; 60: 474-82.

35. Manoharan S, Kolanjiappan K, Suresh K, Panjamurthy K. Lipid peroxidation \& antioxidants status in patients with oral squamous cell carcinoma. Indian J Med Res 2005; 122: 529-34.
36. Meister A, Anderson M.E. Glutathione. Annual Review of Biochemistry. 1983; 52: 711-60.

37. Meuillet E, Stratton S, Prasad CD, Goulet AC, Kagey J, Porterfield B, et al. Chemoprevention of prostate cancer with selenium: an update on current clinical trials and preclinical findings. J. Cell. Biochem 2004; 91: 443-58.

38. Wei WQ, Abnet CC, Qiao YL, Dawsey SM, Dong ZW, Sun XD, et al. Prospective study of serum selenium concentrations and esophageal and gastric cardia cancer, heart disease, stroke, and total death. Am. J. Clin. Nutr 2004; 79: 80-85.

39. Cole-Ezea P, Swan D, Shanley D, Hesketh J. Glutathione peroxidase 4 has a major role in protecting mitochondria from oxidative damage and maintaining oxidative phosphorylation complexes in gut epithelial cells. Free Radic. Biol. Med. 2012; 53: 488-97.

40. Hasegawa T, Okuno T, Nakamuro K, Sayato Y.Identification and metabolism of selenocysteine-glutathione selenyl sulfide (CySeSG) in small intestine of mice orally exposed to selenocysteine. Arch. Toxicol 1996; 71: 39-44. 